\title{
Thermal Expansion Characteristics of Hexaboride Electron Sources and Other Factors Impacting Beam Stability
}

\author{
L. A. Southall,* K. J. Kagarice, ${ }^{*}$ A. M. Thron,* P. M. Canepa, ${ }^{* *}$ and W. A. Mackie* \\ *Applied Physics Technologies, 1600 NE Miller St., McMinnville, OR 97128 USA \\ ** Linfield College, 900 SE Baker St., McMinnville, OR 97128 USA
}

In some electron beam systems it is highly desirable to obtain stable probe current as soon as possible following start-up. For hexaboride cathodes mounted in conventional triode guns, thermal expansion of electron gun components plays a key role in beam stability during this time period. Until thermal equilibrium is established, movement of the emitter relative to other electrode elements alters the shape of the electric fields that determine fundamental parameters of emission area and crossover position. It could be argued that while the common self-biased grid cap system should control total emitted current during thermal expansion, it does not necessarily follow that probe current will also be controlled. Even if the field's zero equipotential lines that define the boundaries of the emitting surface (see Fig.1) are kept fixed by the self-bias as the tip expands forward, subtle changes to the position of the crossover can alter acceptance angle through the anode and thus impact beam current and divergence. The impact of this is dependent on system design and operating parameters.

In this study, the thermal expansion characteristics of several commercially available hexaboride $\left(\mathrm{LaB}_{6}\right.$ and $\left.\mathrm{CeB}_{6}\right)$ cathode designs were examined, both in fixed bias and selfbiased mode. Emission current and probe current were collected for several hours after start-up, and compared against time-tagged video frames of the tip position relative to the grid cap aperture. Through a calibration procedure, it was possible to calculate the position of the tip truncation (emitting plane) within a few microns, and determine the maximum forward expansion as well as the final equilibrium position of the tip. It was found that in self-biased mode, fluctuations in emission current could be fairly well controlled by adjusting system parameters. Probe current fluctuations were more difficult to control via the self-bias circuit, and were found to originate in both alignment perturbation and changes in beam brightness. The maximum forward position of the tip was in close agreement with calculated expansions of the crystal plus support posts, while the final equilibrium position implied a slower forward expansion of the grid cap, as suggested by P. B. Sewell [1].

Thermal expansion characteristics were measured for $\mathrm{LaB}_{6}$ cathodes with three substantially different mount styles (the wire mount, the carbon rod mount and the miniVogel mount) in an Amray 1830 SEM grid cap assembly. A $\mathrm{CeB}_{6}$ mini-Vogel mount was also studied in the Amray cap, as well as in a JEOL JSM 6400 SEM cap, an FEI/Philips 2020 ESEM cap, an FEI TEM cap, and an APTech cap. The results show considerable variation between the cathode/cap combinations, and imply that the extent of thermal expansion and the time required to reach equilibrium could be minimized by careful design and matching of the cathode and grid cap (see Fig. 2). The range of maximum forward expansion of the emitting plane at $1800 \mathrm{~K}$ was 40 to 90 microns from 
its initial room temperature position. The range of final equilibrium position was 20 to 70 microns forward from the room temperature position.

Two other factors impacting beam stability of $\mathrm{LaB}_{6}$ and $\mathrm{CeB}_{6}$ cathodes were also identified and studied: work function changes of the emitting surface in cases of extreme re-contamination by desorbed gases, and periodic emission drop/recovery associated with charging and discharging of the deposited hexaboride/oxide film on the grid cap aperture, as reported by Sewell and Ramachandran [2]. These are of significant importance to cathode/cap design aimed at rapidly achieving and maintaining a stable beam.

\section{References}

[1] P. B. Sewell, Emission Drift - $\mathrm{LaB}_{6}$ and Gun Stability, Kimball Physics, Inc. Tech Bulletin \#LaB 6 -03B, May 1991

[2] P. B. Sewell and K. N. Ramachandran, Grid Aperture Contamination in Electron Guns Using Directly Heated Lanthanum Hexaboride Sources, SEM/1978/Vol.1, 221

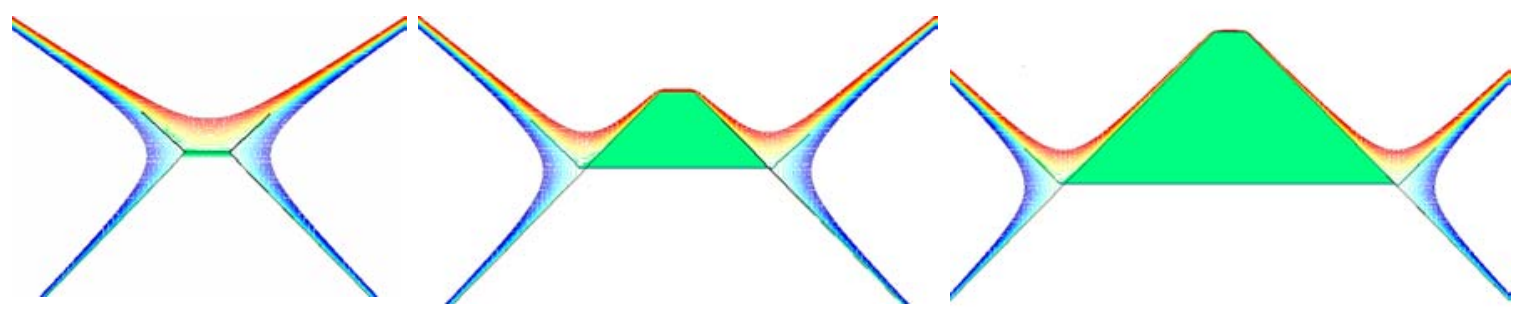

Fig. 1. Field modeling showing progressive enlargement of the emission area (shaded) as the conical tip moves forward a total of 42 microns toward a fixed bias grid cap.

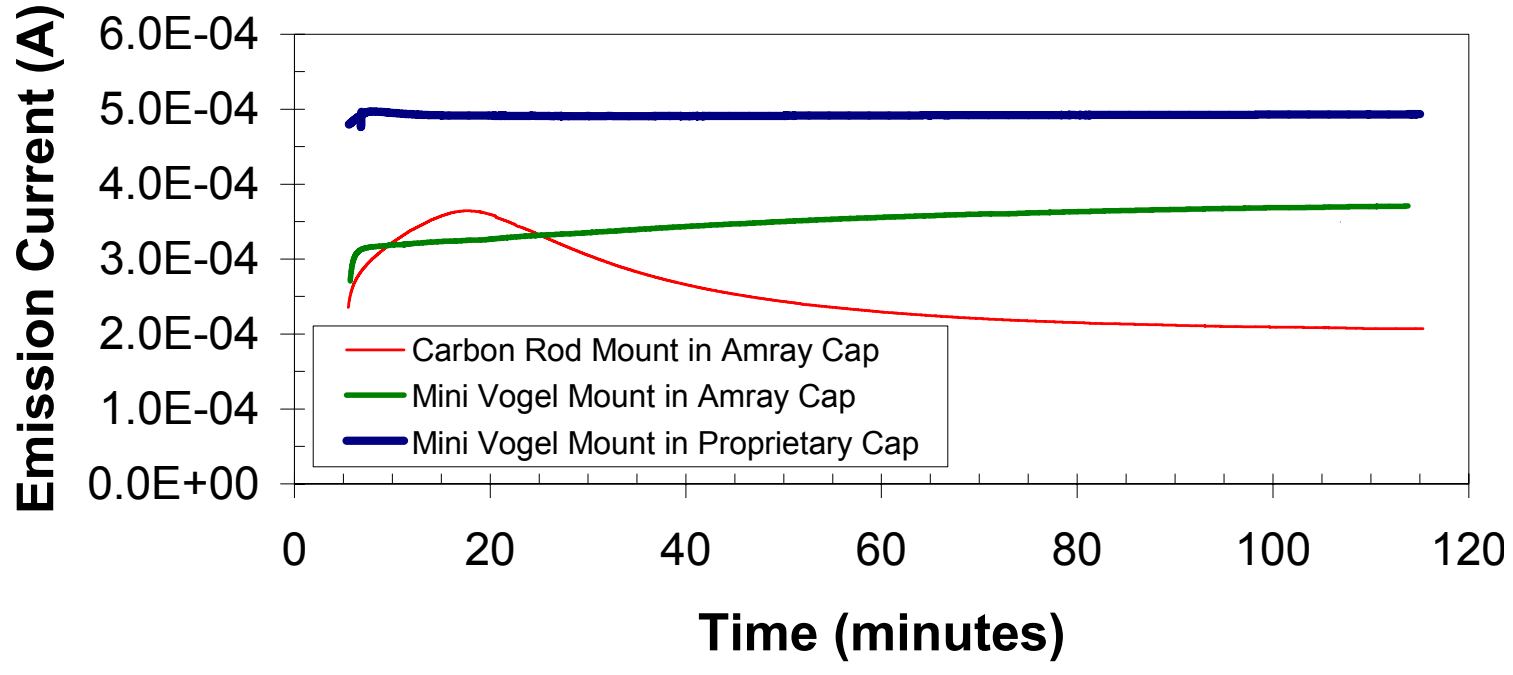

Fig. 2. Comparison of emission current over first 115 minutes after start-up with (3) cathode/cap combinations. Crystal temperature is $1800 \mathrm{~K}$ with fixed bias. 